\title{
The gas has the last laugh
}

\author{
Azad Mashari, MD, FRCPC • Marcin Wasowicz, MD, PhD
}

Received: 29 September 2017/ Accepted: 8 November 2017/Published online: 17 November 2017

(C) Canadian Anesthesiologists' Society 2017

In its long history, nitrous oxide $\left(\mathrm{N}_{2} \mathrm{O}\right)$ has weathered numerous vacillations by practitioners between its popularity and disrepute. ${ }^{1}$ In recent years, there has been a significant decline in its use for surgical anesthesia in high-income countries; nevertheless, globally it remains one of the most commonly used anesthetics. Along with isoflurane and halothane, $\mathrm{N}_{2} \mathrm{O}$ is one of three inhaled anesthetics on the World Health Organization's Essential Medicines List. ${ }^{2}$

In spite of $\mathrm{N}_{2} \mathrm{O}$ 's longevity and a new surge of attention over the past decade, many questions remain about its mechanisms of action and clinical effects. While the large multicentre randomized clinical trial, ENIGMA-II, now backs its safety for major complications, ${ }^{3}$ dose-response relationships for its numerous secondary effects are not well understood. This includes such long-recognized phenomena as analgesia and postoperative nausea and vomiting (PONV).

The study by Mraovic et al. ${ }^{4}$ in this issue of the Journal examined the effect of patients' brief exposure to $70 \% \mathrm{~N}_{2} \mathrm{O}$ in the last $30 \mathrm{~min}$ of laparoscopic-assisted vaginal hysterectomy under isoflurane maintenance. The authors hypothesized that this intervention would lead to more rapid recovery to extubation and orientation without increasing the incidence of PONV. Moreover, they conducted the study even though this was a high-risk procedure in a patient population at high risk for PONV.

A. Mashari, MD, FRCPC $\cdot$ M. Wasowicz, MD, PhD Department of Anesthesia and Pain Management, Toronto General Hospital, Toronto, ON, Canada

A. Mashari, MD, FRCPC - M. Wasowicz, MD, PhD ( $\square)$ Department of Anesthesia, University of Toronto, 200 Elizabeth Street, Eaton North 3-400, Toronto, ON M5G 2C4, Canada e-mail: marcin.wasowicz@uhn.ca
Aside from the intervention, the study largely maintained the existing standards of care at the General Hospital in Zadar, Croatia where it was conducted. Although the study was adequately powered for its primary and secondary outcomes, there was a substantial dropout rate $(\sim 20 \%)$, with only 82 of the 100 randomized patients undergoing the assigned intervention. In all but a single case of protocol violation, this was due to the occurrence of one of the predefined exclusion criteria between the time of randomization (prior to induction of anesthesia) and the time of the intervention.

Several components of the perioperative management protocol in this study may seem obsolete or not understandable to practitioners in high-income countries. These include the routine use of isoflurane for relatively short surgery, the absence of routine PONV prophylaxis in a high-risk setting, and the use of thiopental, meperidine, and metoclopramide as first-line agents. While these aspects may seemingly limit the clinical relevance of the study in certain settings, in our view, the study and its findings have significant value for several reasons. First, the majority of the apparently obsolete elements are in fact routinely used in most middle- and low-income countries (comprising nearly half of global surgical procedures) ${ }^{5}$ where newer agents are unavailable, prohibitively expensive, or not cost effective based on current evidence. In fact, the protocol used in this study is more sophisticated than that available to many practitioners in most low-income countries, where the use of halothane remains the standard and often the only volatile anesthetic besides $\mathrm{N}_{2} \mathrm{O}$. Therefore, the study has clear clinical relevance for practitioners working in these settings, practitioners whose clinical concerns and patient populations are often poorly reflected in major international medical journals. ${ }^{6}$ 
Second, the study has significant relevance, even for practitioners with routine access to newer volatile anesthetics. While the availability of generic versions of these agents has reduced the cost differential compared with isoflurane, they still remain substantially (five to eight times) more expensive than isoflurane in most countries. ${ }^{7,8}$ Significant cost savings have been documented through educational initiatives that have encouraged an increased use of isoflurane. ${ }^{7}$ The shift away from isoflurane in many high-income countries has not been based on evidence of clinical superiority of the newer agents. Where data are available, for example in cardiac surgery, there appears to be no advantage to the use of more costly agents. ${ }^{8}$ Isoflurane is likely still the primary volatile anesthetic used for cardiac surgery in the United Kingdom. ${ }^{9}$ For short surgeries, use of the newer agents is often justified on the grounds of earlier recovery to extubation, which is generally on the order of two to four minutes, ${ }^{10}$ a reduction comparable to that found by Mraovic et al. Furthermore, consistent with several other small studies, fewer patients in the $\mathrm{N}_{2} \mathrm{O}$ group required postoperative opioids in the first two hours, and in fact, the incidence of treatment for PONV was decreased in the $\mathrm{N}_{2} \mathrm{O}$ group.

While limited, the available literature suggests that the dose-response relationship of $\mathrm{N}_{2} \mathrm{O}$ for both analgesia and PONV is significantly nonlinear. Pro-emetic effects do not seem to appear until exposure exceeds approximately 45 min, while the analgesic effects appear to peak at around 30 minutes and decline thereafter. The relationship between duration and concentration of exposure remains to be studied. ${ }^{1}$

In conclusion, the study findings suggest that a simple, low-cost, and widely available intervention has multiple desirable effects-i.e., more rapid recovery, potentially reduced PONV, and potentially improved analgesia. If these findings withstand the test of time, their clinical impact can be substantial, especially in low-resource settings. We applaud the authors for surmounting the numerous additional obstacles that commonly bedevil clinical research in the absence of the financial and institutional resources often available in high-income countries. We also applaud the Canadian Journal of Anesthesia for its editorial commitment to global health ${ }^{11}$ and for taking steps to address the underrepresentation of low- and middle-income countries in international medical journals.

Nitrous oxide appears to have weathered yet another storm. After one and a half centuries of clinical use, it continues to raise questions for yet another generation of researchers and practitioners whom it may well outlast!

\section{Rira bien le gaz qui rira le dernier}

Au cours de sa longue histoire, le protoxyde d'azote $\left(\mathrm{N}_{2} \mathrm{O}\right)$ a navigué entre les nombreuses hésitations des praticiens, oscillant entre popularité et mauvaise réputation. ${ }^{1}$ Ces dernières années, son utilisation pour l'anesthésie chirurgicale a subi un déclin important dans les pays à revenu élevé; cependant, cet agent demeure l'un des anesthésiques les plus fréquemment utilisés dans le monde. Avec l'isoflurane et l'halothane, le $\mathrm{N}_{2} \mathrm{O}$ est l'un des trois anesthésiques par inhalation figurant sur la Liste de médicaments essentiels de l'Organisation mondiale de la santé. ${ }^{2}$

En dépit de la longévité du $\mathrm{N}_{2} \mathrm{O}$ et du regain d'intérêt pour cet agent observé depuis une dizaine d'années, de nombreuses questions demeurent en suspens quant à ses mécanismes d'action et ses effets cliniques. Alors que l'étude clinique randomisée multicentrique d'envergure ENIGMA-II démontrait son innocuité en cas de complications majeures, ${ }^{3}$ les relations de dose-réponse pour ses nombreux effets secondaires demeurent mal comprises. Ces effets comprennent des phénomènes connus depuis longtemps, tels que l'analgésie et les nausées et vomissements postopératoires (NVPO).

L'étude de Mraovic et coll., ${ }^{4}$ publiée dans ce numéro du Journal, a examiné l'effet d'une brève exposition des patientes de l'étude à du $\mathrm{N}_{2} \mathrm{O} 70 \%$ au cours des 30 dernières minutes d'une hystérectomie vaginale par laparoscopie réalisée sous isoflurane. Les auteurs ont émis l'hypothèse que cette intervention entraînerait une récupération plus rapide jusqu'à l'extubation et l'orientation et ce, sans augmenter l'incidence de NVPO. En outre, ils ont réalisé l'étude en dépit du fait qu'il s'agissait d'une intervention à risque élevée auprès d'une population courant un risque élevé de NVPO. Hormis cette intervention, l'étude a maintenu, en grande partie, les normes existantes de soins de l'Hôpital général de Zadar, en Croatie, où elle a été réalisée. Bien que l'étude disposait de la puissance nécessaire pour donner réponse à ses critères d'évaluation primaire et secondaires, le taux d'abandon a été considérable ( $\sim 20 \%)$ : en effet, seules 82 des 100 patientes randomisées ont subi l'intervention assignée. Dans tous les cas, hormis un cas de violation du protocole, l'abandon de l'étude s'est justifié par la survenue de l'un des critères d'exclusion prédéterminés entre le moment de l'allocation aux groupes (avant l'induction de l'anesthésie) et le moment de l'intervention.

Plusieurs composantes du protocole de prise en charge périopératoire de cette étude pourraient sembler obsolètes 
et incompréhensibles aux praticiens provenant de pays à revenu élevé. Par exemple, citons l'utilisation de routine de l'isoflurane pour une chirurgie relativement courte, l'absence d'une prophylaxie de routine contre les NVPO dans un contexte à risque élevé, et l'utilisation de thiopental, de mépéridine et de métoclopramide comme agents pharmaceutiques initiaux. Alors que ces aspects pourraient donner l'illusion de circonscrire la pertinence clinique de cette étude à certains contextes, selon nous, l'étude et ses résultats possèdent une importante valeur intrinsèque pour plusieurs raisons. En premier lieu, la majorité des composantes apparemment obsolètes de cette intervention sont en fait régulièrement utilisées dans la plupart des pays à revenu faible ou intermédiaire (ce qui englobe près de la moitié des interventions chirurgicales dans le monde), ${ }^{5}$ dans lesquels les agents plus récents ne sont pas disponibles, ou sont disponibles à un prix prohibitif, ou sont non rentables selon les données probantes actuelles. En fait, le protocole utilisé dans cette étude est plus sophistiqué que ce qui est disponible pour bon nombre de praticiens dans la plupart des pays à revenu faible, dans lesquels l'utilisation d'halothane constitue toujours la norme et est souvent le seul agent anesthésique par inhalation hormis le $\mathrm{N}_{2} \mathrm{O}$. Ainsi, l'étude fait preuve d'une pertinence clinique évidente pour les praticiens travaillant dans ces conditions, des praticiens dont les préoccupations cliniques et populations de patients sont souvent peu représentées dans les revues médicales internationales majeures. $^{6}$

Deuxièmement, l'étude est importante même pour les praticiens ayant facilement accès aux nouveaux anesthésiques par inhalation. Alors que la disponibilité de versions génériques de ces agents a réduit la différence de coût par rapport à l'isoflurane, dans la plupart des pays, ils demeurent tout de même considérablement (cinq à huit fois) plus onéreux que l'isoflurane. ${ }^{7,8}$ D'importantes économies ont été documentées grâce à des initiatives éducationnelles encourageant l'utilisation accrue d'isoflurane. ${ }^{7}$ Dans plusieurs pays à revenu élevé, le déclin de l'utilisation de l'isoflurane ne s'est pas appuyé sur des données probantes attestant de la supériorité clinique de nouveaux agents. Là où les données sont disponibles, comme par exemple en chirurgie cardiaque, il semble n'y avoir aucun avantage à utiliser des agents plus onéreux. ${ }^{8} \mathrm{Au}$ Royaume-Uni par exemple, l'isoflurane est probablement toujours l'anesthésique par inhalation de choix en chirurgie cardiaque. ${ }^{9}$ Pour les chirurgies de courte durée, l'utilisation d'agents plus récents se justifie souvent par une récupération précoce jusqu'à l'extubation, qui est en général de l'ordre de deux à quatre minutes, ${ }^{10}$ soit une réduction comparable à celle observée par Mraovic et coll.
En outre, tout comme plusieurs autres petites études l'ont observé, un nombre moindre de patientes du groupe $\mathrm{N}_{2} \mathrm{O}$ ont nécessité des opiö̈des au cours des deux premières heures postopératoires et, en fait, l'incidence de traitement pour les NVPO était réduite dans ce groupe.

Bien que limitée, la littérature disponible suggère que la relation de dose-réponse du $\mathrm{N}_{2} \mathrm{O}$ tant pour l'analgésie que pour les NVPO est significativement non linéaire. Les effets pro-émétiques ne semblent pas survenir avant que l'exposition à l'agent ne dépasse environ 45 minutes, alors que les effets analgésiques semblent atteindre leur point culminant vers 30 minutes d'exposition, puis décliner par la suite. La relation entre la durée et la concentration de l'exposition devra faire l'objet d'autres études. ${ }^{1}$

En conclusion, selon les résultats observés dans cette étude, une intervention simple, peu coûteuse et facilement accessible semble avoir de nombreux effets désirables soit une récupération plus rapide, des NVPO potentiellement réduits, et une analgésie potentiellement améliorée. Si ces résultats résistent à l'épreuve du temps, leur impact clinique pourrait être considérable et ce, particulièrement dans les contextes où les ressources sont très limitées. Nous félicitons les auteurs d'avoir surmonté les nombreux obstacles supplémentaires qui minent fréquemment la recherche clinique en l'absence des ressources financières et institutionnelles souvent disponibles dans les pays à revenu élevé. Nous félicitons également le Journal canadien d'anesthésie pour son engagement rédactionnel envers la santé mondiale ${ }^{11}$ et pour ses actions visant à pallier la sous-représentation des pays à revenu faible et moyen dans les revues médicales internationales.

Le protoxyde d'azote semble avoir survécu à une autre tempête. Après un siècle et demi d'utilisation clinique, il continue de soulever des questions pour une nouvelle génération de chercheurs et de praticiens, auxquels il pourrait bien survivre!

\section{Conflicts of interest None declared.}

Editorial responsibility This submission was handled by Dr. Philip M. Jones, Associate Editor, Canadian Journal of Anesthesia.

Funding Merit Award, Department of Anesthesia, University of Toronto (MW).

\section{Conflit d'intérêt Aucun.}

Responsabilité éditoriale Cet article a été traité par Dr Philip M. Jones, rédacteur adjoint, Journal canadien d'anesthésie.

Financement Bourse de mérite, département d'anesthésie, Université de Toronto (MW). 


\section{References}

1. Sanders RD, Weimann J, Maze M. Biologic effects of nitrous oxide: a mechanistic and toxicologic review. Anesthesiology 2008; 109: 707-22.

2. World Health Organization. WHO Model List of Essential Medicines 19th List - April 2015, Amended November 2015. Available from URL: http://www.who.int/medicines/publica tions/essentialmedicines/EML_2015_FINAL_amended_NOV2015. pdf?ua=1 (accessed October 2017).

3. Myles PS, Leslie K, Chan MTV, et al. The safety of addition of nitrous oxide to general anaesthesia in at-risk patients having major non-cardiac surgery (ENIGMA-II): a randomised, singleblind trial. Lancet 2014; 384: 1446-54.

4. Mraovic B, Simurina T, Gan TJ. Nitrous oxide added at the end of isoflurane anesthesia hastens early recovery without increasing the risk for postoperative nausea and vomiting - a randomized clinical trial. Can J Anesth 2018; 65: this issue. http://doi.org/10. 1007/s12630-017-1013-y

5. Weiser TG, Haynes AB, Molina $G$, et al. Size and distribution of the global volume of surgery in 2012. Bull World Health Organ 2016; 94: 201-9F.
6. Rochon PA, Mashari A, Cohen A, et al. Relation between randomized controlled trials published in leading general medical journals and the global burden of disease. Can Med Assoc J 2004; 170: 1673-7.

7. Miller SA, Aschenbrenner CA, Traunero JR, et al. $\$ 1.8$ Million and counting: how volatile agent education has decreased our spending $\$ 1000$ per day. J Clin Anesth 2016; 35: 253-8.

8. Jones PM, Bainbridge D, Chu $M W$, et al. Comparison of isoflurane and sevoflurane in cardiac surgery: a randomized noninferiority comparative effectiveness trial. Can J Anesth 2016; 63: 1128-39.

9. Charlesworth M, Ashworth A, Stirling S. Isoflurane use is not associated with prolonged intensive care unit stay following routine cardiac surgery when compared to sevoflurane. Can J Anesth 2017; 64: 100-1.

10. Dexter F, Tinker JH. Comparisons between desflurane and isoflurane or propofol on time to following commands and time to discharge. A metaanalysis. Anesthesiology 1995; 83: 77-82.

11. Winker MA, Ferris LE; World Association of Medical Editors (WAME) Ethics and Policy Committee, and the WAME Board. Promoting global health: the World Association of Medical Editors' position on editors' responsibility. Indian J Urol 2015; 31: 165-7. 